Artículo de INVESTIGACIÓN

Recibido: 9 de septiembre de 2020. Aprobado: 12 de abril de 2021.

DOI: $10.17151 /$ rasv.2021.23.2.8

\section{"Plantas que toda la vida han estado" "1: una co-investigación alrededor de la cocina y las relaciones bioculturales asociadas a plantas alimenticias locales en la ruralidad de Bogotá}

"Plants that have always been there": A co-research around cooking and biocultural relationships associated with local food plants in rural Bogotá

\section{Resumen}

A través de un proceso de co-investigación con sabedores de la ruralidad bogotana, se documentaron prácticas culinarias tradicionales y se realizaron encuentros para conversar sobre sus implicaciones territoriales y los desafíos de sus transformaciones. El diálogo permitió reactivar la memoria y las percepciones que, desde la cocina, remiten al territorio, y conversar sobre los riesgos y potencialidades de las innovaciones en las prácticas suscitadas, considerando la porosidad de las fronteras urbano-rurales. Se evidencia una persistencia de conocimientos y relaciones con la biodiversidad local, que representan un acervo esencial para pensar la conservación y dinamización

Cómo citar este artículo:

Consuegra, C., Ortiz, S., Cely-Santos, M., van der Hammen, M. C. y Pérez, D. (2021). "Plantas que toda la vida han estado": una co-investigación alrededor de la cocina y las relaciones bioculturales asociadas a plantas alimenticias locales en la ruralidad de Bogotá Revista de Antropología y Sociología: Virajes, 23(2), 163-185. https://doi.org/10.17151/rasv.2021.23.2.8

\section{Cristina Consuegra}

Investigadora Independiente, Bogotá, Colombia.

$\square$ cristinaconsuegra@gmail.com (D) ORCID: 0000-0003-3290-5165

ज Google Scholar

Stefan Ortiz

Universidad Leuphana de Lüneburg, Alemania.

Universidad Nacional de

Colombia, Bogotá, Colombia.

$\square$ sortizp@unal.edu.co

(1) ORCID: 0000-0002-8927-8636

sc Google Scholar

Marcela Cely-Santos

Rachel Carson Center for the

Environment and Society,

Múnich, Alemania.

$\square$ cely.sandramarcela@gmail.com

DD ORCID: 0000-0001-9792-8793

sc Google Scholar

María Clara

VAN DER HAMmEN

Universidad Externado de

Colombia, Bogotá, Colombia.

v mariaclaravanderhammen@

hotmail.com

(1) ORCID: 0000-0001-9623-6875

$\triangle$ Google Scholar

Darío Pérez

Universidad de Montpellier,

Francia. PALOC, Muséum

National d'Histoire Naturelle,

IRD, Paris, Francia). Universidad

Nacional de Colombia (GIMBBE,

Instituto de Ciencias Naturales).

$\square$ daaperezgo@unal.edu.co

(1) ORCID: 0000-0002-8664-1321

^n Google Scholar 
de la diversidad biocultural y su integración como parte del patrimonio cultural material e inmaterial de Bogotá.

Palabras clave: Memoria y diversidad biocultural, Cocina tradicional, Ciencia participativa, Conocimientos locales.

\section{ABstract}

A process of co-research with farmers in the rural area of Bogotá, documented traditional culinary practices and held meetings to discuss their territorial implications and the challenges of their transformations. The dialogues promoted the reactivation of memories and perceptions, linking the space of kitchens and territories, and included conversations on the risks and opportunities of innovations triggered by urban-rural relations. This research sheds light on the persistence of linkages related to local biodiversity, which represent a key asset for the conservation of biocultural diversity and of the material and immaterial heritage of Bogota.

Keywords: Biocultural memory and diversity, Traditional cuisine, Participatory research, Local knowledge.

\section{Introducción}

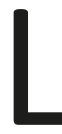

as prácticas relacionadas con producir, preparar y compartir el alimento son parte de las relaciones bioculturales que contribuyen a la construcción social de los territorios. El repertorio de vínculos entre un grupo humano y su entorno, enmarcado en las cosmovisiones, conocimientos y prácticas que emergen de la relación histórica entre las personas y los ecosistemas (Toledo y Barrera-Bassols, 2014), se construye y mantiene en comunidad, siendo influenciado por el contexto social y ecológico en que se desarrolla. El carácter colectivo y sistémico de esas relaciones alrededor de la alimentación implica que esta está en continua transformación y negociación mediante intercambios y diálogos entre conocimientos, significados, símbolos y prácticas a distintas escalas que se reflejan en los procesos locales. Esto incluye las tendencias de las relaciones urbano-rurales y locales-globales (Bowman y Zilberman, 2013).

La homogeneización cultural a la que tienden los discursos y prácticas de la globalización ha afectado negativamente la diversidad de relaciones bioculturales que subyacen a las prácticas alimentarias, precipitando su simplificación y la erosión de la biodiversidad que los sustenta 
(Ramirez, 2007). La expansión de la agricultura industrial basada en monocultivos, cuyo discurso se basa en la necesidad de producir mayores cantidades de alimentos para una población creciente, ha acelerado la simplificación de los sistemas productivos agrícolas mediante la introducción de tecnologías e insumos externos que generan mayor dependencia, desconocen los conocimientos locales (Benz et al., 2000; Voeks y Leony, 2004; Reyes-García et al., 2005) y promueven la pérdida de biodiversidad (Bonilla-Bolaños y Singaña-Tapia, 2019). Así, especialmente en territorios densamente poblados y urbanizados, las relaciones desiguales en la creación, la transferencia y la apropiación de conocimientos, tecnologías y prácticas agrícolas ligadas a los sistemas agroalimentarios, se encuentran en constante tensión en cada uno de sus eslabones.

El caso del abasto alimentario en Bogotá es particular, considerando que el $77 \%$ del territorio administrativo de esta capital nacional es rural. Las comunidades campesinas bogotanas son alrededor del $1 \%$ de la población total, y conviven con ecosistemas importantes de la alta montaña andina. Estos, a su vez, hacen parte del páramo más grande del mundo (Sumapaz), tienen múltiples lagunas y quebradas, y también comprenden el valle del río Tunjuelo, que recorre la ruralidad de Usme y Ciudad Bolívar antes de entrar a la ciudad (Ortiz et al., 2017). A pesar de que en esta zona se produce una parte de los alimentos que se consumen en el área urbana, la ruralidad de Bogotá ha sido marginalizada en los procesos de planeación, reflexión y construcción de política pública. La demanda por ciertos alimentos en los mercados de la ciudad ha promovido el establecimiento de monocultivos, principalmente de papa, arveja y habas, relegando la diversidad de hortalizas, tubérculos, especies medicinales y aromáticas, y especies silvestres propias de los ecosistemas altoandinos.

En territorios situados en la frontera urbano-rural, como es el caso de este estudio, los procesos de urbanización suelen generar presiones directas sobre las relaciones bioculturales de la ruralidad, transformando no sólo los espacios físicos, sino también las prácticas agrícolas que deben adaptarse en función de lo que la ciudad pide de ellos para expandirse (Pérez-Martínez, 2011). Este no es un caso aislado, en los últimos años los conocimientos asociados a una gran diversidad de plantas que han sustentado formas de ver y conocer el mundo se están erosionando a tasas alarmantes a escala global por efecto de la industrialización agrícola (Laurance et al., 2006; Ellis et al., 2012; Vandebroek y Voeks, 2019). Este fenómeno, para el caso particular de la producción de alimentos en Bogotá, reduce el acceso a dietas diversas y a sus nutrientes, y simplifica el repertorio agronómico y culinario de poblaciones enteras, 
homogeneizando los espacios y las prácticas que conforman los circuitos agroalimentarios (Fu et al., 2010; Montenegro de Wit, 2016; Wolff, 2004).

Los paisajes rurales de Bogotá, donde aún se desarrollan prácticas tradicionales de manejo agrícola diversificado, suelen ser refugios de altos niveles de biodiversidad e importantes repositorios de conocimiento ecológico local (Zimmerer, 2004; Clavijo-Ponce y Pérez-Martínez, 2014; Pérez y Matiz-Guerra, 2017) que conforman sistemas socioecológicos con un robusto patrimonio biocultural, caracterizados por las relaciones de conocimientos, prácticas y experiencias que involucran a diversas especies, y permiten una agricultura multifuncional que posibilita la innovación local y la adaptación al cambio socioambiental (Nazarea-Sandoval, 1998; Perreault, 2005; Perrault-Archamault y Coomes, 2008; Rhoades y Nazarea, 1999; Thrupp, 2002). Estos sistemas pueden motivar la transformación del sistema agroalimentario basado en el monocultivo y el uso intensivo de insumos externos, por lo que reconocer sus visiones del mundo y reivindicar sus experiencias es clave para trazar nuevos caminos hacia la sostenibilidad (Andrade et al., 2018).

Promover los paisajes rurales que sustentan el bienestar socioecológico de las relaciones campo-ciudad requiere de aproximaciones investigativas innovadoras e inclusivas. Existen reportes en donde se evidencian avances en ese sentido, enfocados en explorar las causas de la erosión del conocimiento ecológico tradicional (Gómez-Baggethun, 2009), o los cambios y tendencias recíprocas de la diversidad biológica y cultural (Reyes-García et al., 2013; Tsuji, 1996). Estos resultados brindan un panorama sobre las acciones necesarias para proteger la diversidad biológica y el conocimiento ecológico tradicional. Sin embargo, también es importante entender el rol del patrimonio biocultural como fuente de la capacidad adaptativa de los sistemas socioecológicos, y de las relaciones y valoraciones implicadas en sus experiencias. Para esto se requiere un trabajo horizontal con las comunidades locales donde se reconozcan, dinamicen y documenten los saberes locales basados en experiencias situadas y cotidianas (Barthel et al., 2013), y se creen espacios para evidenciar tanto las vulnerabilidades de las comunidades locales, como sus oportunidades de adaptación. Lo anterior es posible mediante estrategias de co-investigación que se fundamenten en la participación, el diálogo y el aprendizaje horizontal involucrando y reivindicando sistemas de conocimientos diversos. En este sentido, la ciencia participativa y la co-investigación son herramientas de inclusión que permiten establecer puentes entre distintas formas de conocer y experimentar el entorno, por ejemplo, entre la ciencia convencional, generalmente eurocéntrica, y las epistemologías situadas, cotidianas y experienciales de las comunidades 
rurales (Fals-Borda, 1999; Polk, 2015). Las metodologías participativas reconocen el potencial del diálogo de conocimientos para contribuir a la solución de problemas y a la transformación social (Delgado y Escobar, 2006; Harding, 1994; Haraway, 2003).

En este estudio presentamos un proceso de co-creación de conocimiento alrededor de los sistemas agroalimentarios de la Bogotá rural. A través de un proceso de ciencia participativa que incluyó la recolección de información primaria por parte de las comunidades campesinas de la ruralidad de Bogotá y la organización de encuentros culinarios, se promovió un diálogo horizontal que permitió 1) activar la memoria sobre algunas plantas alimenticias locales, 2) documentar prácticas culinarias cotidianas e innovadoras, y 3) dialogar sobre la recuperación y la apropiación de conocimiento en respuesta a las transformaciones sociales y ambientales que amenazan la existencia de los sistemas bioculturales en un contexto urbano-rural.

\section{Metodología}

En una primera fase, realizamos una convocatoria local acudiendo a los habitantes de la ruralidad de Bogotá con quienes existían antecedentes de trabajo investigativo. Esto se desarrolló en las localidades de Usme y Ciudad Bolívar, que se localizan en la cuenca media y alta del río Tunjuelo (Figura 1). Como resultado, vinculamos a un grupo de jóvenes interesados en desarrollar un ejercicio de ciencia participativa a partir de preguntas y necesidades propias. El grupo de co-investigadores incluyó a dos mujeres y un hombre, los tres pertenecientes a las comunidades campesinas de la ruralidad, y a una mujer habitante de la Bogotá urbana con experiencia en el manejo de huertas urbanas y la conservación de semillas locales. 

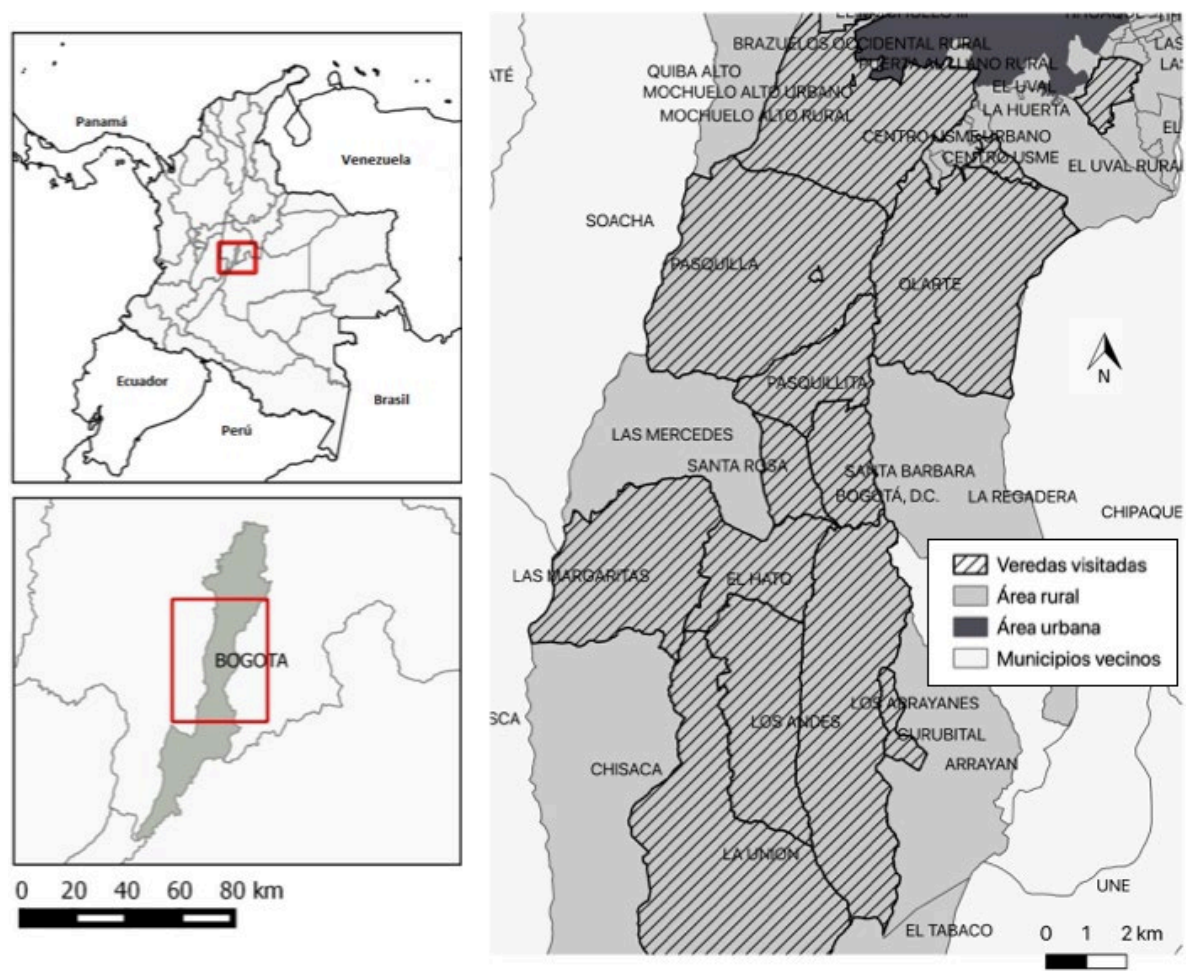

$0 \quad 20 \quad 40 \quad 60 \quad 80 \mathrm{~km}$

Figura 1. Área de estudio.

Fuente: elaboración propia.

Junto con las y los co-investigadores, diseñamos preguntas y formatos para obtener información primaria, buscando generar y divulgar los conocimientos y diagnósticos de problemas que ellas y ellos mismos han podido acumular con base en la experimentación y observación cotidiana. Adicionalmente, hicimos un mapa de actores para determinar las y los sabedores a consultar, y formulamos un cronograma conjunto de trabajo en campo. Además, las y los participantes de la academia realizamos un recorrido por 17 veredas de la ruralidad de Bogotá, recolectando la información primaria a partir de entrevistas semiestructuradas con 47 habitantes de la ruralidad. En las entrevistas indagamos acerca del uso alimenticio de las plantas disponibles en el territorio, haciendo un especial énfasis en las recetas locales que recordaban.

La vinculación de co-investigadores provenientes de la zona de estudio y de la ciudad fue una oportunidad para enriquecer el intercambio de experiencias y percepciones sobre el proceso de investigación, sobre las problemáticas de la alimentación en la ruralidad de Bogotá, y sobre los conflictos en la relación urbano-rural. Este proceso implicó también aprendizajes y acciones de apoyo mutuo, definición conjunta de preguntas 
de investigación, la toma consensuada de decisiones y, en general, un proceso horizontal de aproximación al territorio y a la temática de estudio.

Además de participar en la investigación sobre las especies priorizadas, las y los co-investigadores iniciaron proyectos investigativos personales y autónomos (Tabla 1); esto permitió que hubiera una mayor comprensión del método científico convencional y un posicionamiento crítico desde la experiencia personal. El apoyo incluyó la formulación conjunta de los objetivos, resultados esperados y la metodología de trabajo sugerida para obtener datos.

Tabla 1. Investigaciones sobre plantas silvestres formuladas por los coinvestigadores

\begin{tabular}{ll}
\hline Investigador & Tema propuesto \\
\hline Erika & $\begin{array}{l}\text { Transmisión de conocimientos y formas de apropiación de los mismos. Como } \\
\text { producto relacionado a este tema, Erika trabajó en una compilación de historias } \\
\\
\text { sobre las cinco plantas y sus usos respectivos, las cuales estarán narradas por los } \\
\text { niños de } 3,4 \text { y } 5 \text { grado de la escuela de la vereda Pasquillita. }\end{array}$ \\
\hline Adriana & $\begin{array}{l}\text { Nuevos usos culinarios para el morón de páramo (Rubus spp.). El producto de } \\
\text { Adriana comprende la invención de una receta de yogurt de morón, la cual incluye } \\
\text { una etiqueta narrativa sobre el morón y la documentación de todas las fases del } \\
\text { proceso de invención. }\end{array}$ \\
\hline Usodrigo y manejo agroecológico de las huertas. Rodrigo hizo una compilación basada \\
en cinco ejemplos de arreglos agroecológicos que permitan evitar el uso de agro- \\
químicos en las huertas familiares de la ruralidad. \\
\hline Preparaciones tradicionales e innovaciones culinarias que incentiven el consumo \\
de las especies que crecen en el territorio y están en riesgo de desaparecer. El obje- \\
tivo de Zonia fue documentar estas recetas y producir una cartilla para difundirlas.
\end{tabular}

Fuente: elaboración propia.

Posteriormente, facilitamos dos encuentros culinarios concebidos como espacios de diálogo, reunión e intercambio para reactivar y recrear la memoria asociada a las tradiciones culinarias de los habitantes de la cuenca media y alta del río Tunjuelo que habían sido mencionadas en las entrevistas. En estos encuentros participaron las y los miembros de las comunidades campesinas de la ruralidad de Bogotá, y dos cocineras tradicionales. En estos espacios se conversó sobre las tradiciones culinarias locales y la diversidad agroalimentaria. Además, hubo espacio para promover la innovación, la creatividad, la reapropiación y la resignificación de las formas en que la comunidad se relaciona con los alimentos tradicionales mediante las recetas preparadas (Consuegra et al., 2017). El desarrollo de estos encuentros fue gestionado por las y los co-investigadores en dos momentos: el primero, liderado por las cocineras de la cuenca media y alta del río Tunjuelo, en el que se identificaron 
y desarrollaron diferentes preparaciones de la Bogotá rural, y el segundo, liderado por dos cocineras invitadas desde otras regiones del país: Isabel Guevara, cocinera tradicional de Nariño, Colombia, agricultora urbana en Bogotá y guardiana de semillas criollas y nativas, y de Jennifer Rodríguez, dueña y cocinera del restaurante Mestizo Cocina de Origen $₫$ en Mesitas del Colegio, Cundinamarca, Colombia. La participación de la comunidad fue activa y espontánea.

Para enfatizar los sentidos de pertenencia y reciprocidad, se coordinó con las comunidades y los investigadores los aportes, de manera que todos fueron anfitriones y comensales a la vez. Ambos encuentros incluyeron recetas diversas, lo que permitió que los participantes escogieran espontáneamente unirse a cualquier preparación. La realización fue precedida por una exposición de ingredientes y un intercambio de conocimientos sobre ellos (Figura 2).

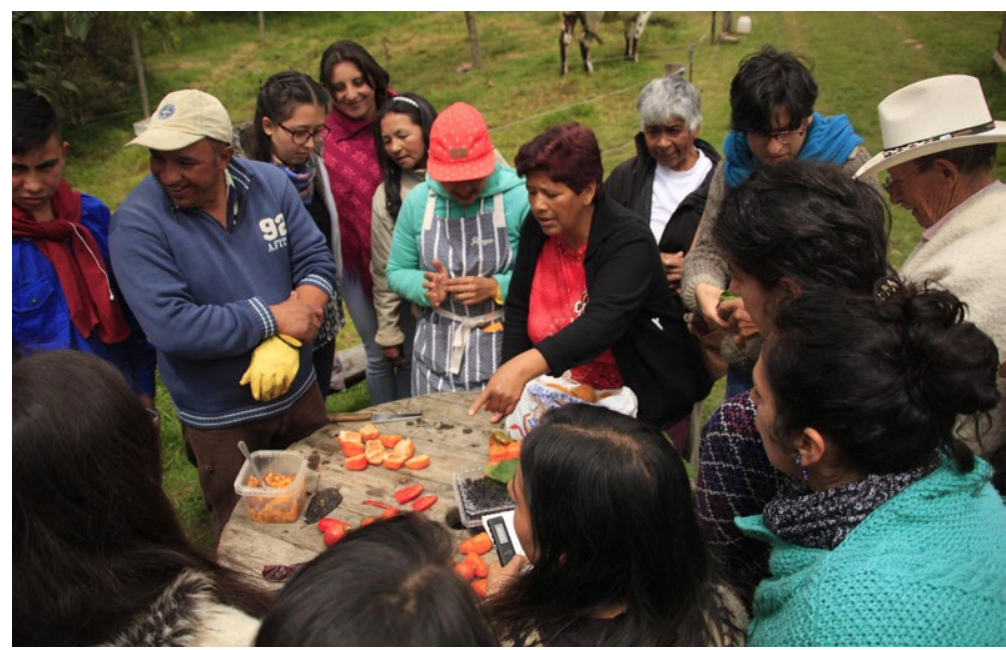

Figura 2. Ajíes sobre la mesa, vereda El Hato, Usme. Fuente: foto tomada por Gerrit Stollbrock.

Resultados y discusión

\section{a. La memoria acerca de las plantas alimenticias locales}

Diariamente los habitantes de la ruralidad de Bogotá dan vida al conocimiento y prácticas locales sobre las plantas de la región, y su memoria está cargada de experiencias transmitidas a través de las generaciones. Así, los conocimientos resultantes sobre las plantas son una mezcla de recuerdos de los aprendizajes individuales y colectivos derivados de su vida como habitantes de la montaña (Pérez y Matiz-Guerra, 2017). 
Pues, la verdad pues yo lo miraba como algo que sí, como para uno, que uno puede usar. Así puede ser con la de papayo, con la de tomate. Uno tiene, para que pueda servir harto. Uno lo mira ya como una matica de jardín, como algo de adorno más que todo. A veces la podo y la arreglo, me gusta verla así. Porque ella queda toda amarillita. Uno la ve como algo, como un jardín tal vez. La costumbre de sembrar. Tal vez uno no intenta otras cosas... Ahorita pues no la he cuidado, pero sí, la miro como, puede decirse como una reliquia porque por acá de esta especie ya no se consigue. (Entrevista a habitante de la vereda Chiguaza, agosto de 2018)

Las relaciones entre las plantas alimenticias locales y la comunidad aún persisten, aunque el conocimiento asociado se ha transformado $u$ olvidado a lo largo del tiempo. Estas plantas tenían varios usos que iban desde el consumo como alimento hasta su integración como elementos en los juegos cotidianos de infancia.

Yo me ponía a jugar con eso, con las pepitas del mortiño, al restaurante. A echar unas pepitas ahí en un plato, con ollas, y que vendía uno eso. Con mi hermana jugábamos con las vecinas (...) Pagábamos con las mismas hojas, como si fueran los billetes (...) Con las hojas y pepas de la uva y las hojas del mortiño como si fueran moneditas. (Entrevista a habitante de la vereda Santa Rosa, septiembre de 2018)

El mortiño me recuerda mi niñez cuando estábamos todos acá, todos en familia y había esos frutos y todo el mundo a ir a recoger mortiños en bolsitas, eso sí era cada uno con su bolsita a ver cuál recogía más. Eso era el plan, sí. Y bueno, si era necesario, "vámonos" hasta la finca del vecino y si había hartas, bueno "quién es el que se va a subir allá" (...). (Entrevista a habitante de la vereda Andes, agosto de 2018)

Según las entrevistas, varias de las especies, como el "mortiño" (Hesperomeles goudotiana (Decne.) Killip) y la "uva camarona" (Macleania rupestris (Kunth) A.C.Sm.), han conservado su estado silvestre (Ortiz et al., 2021) y otras se cultivan para el autoconsumo y para intercambios locales, aún sin fines comerciales. Este es el caso de algunas variedades locales de papa (Solanum phureja Juz. \& Bukasov), del "ají criollo" (Capsicum pubescens L.), o de la "guatila" (Sechium edule (Jacq.) Sw.).

Aunque la recolección de las plantas en estado silvestre se ha reducido, según indican las y los sabedores consultados, aún su consumo se mantiene a escala local y no se ha extendido debido a que no representan una fuente de ingreso de dinero porque no tienen demanda en 
el mercado. Las relaciones que las comunidades tienen con las plantas de la región son influenciadas por ciertas dinámicas socioeconómicas que regulan la producción, acceso y el consumo de alimentos (TorresPabón, 2020; Ortiz et al., 2021); por ejemplo, los procesos de migración urbano-rural han fracturado los procesos de transmisión oral del conocimiento. La reducción de zonas de contacto -material e inmaterial- entre los campesinos y la biodiversidad se refleja en la erosión de los vínculos y conocimientos asociados (Cabrera-Serrano, 2005). Sin embargo, aún se conserva un amplio conocimiento etnobotánico acerca de los usos de las plantas, evidenciado en el reconocimiento de gran diversidad de nombres comunes (Rodríguez-Calderón et al., 2019) y la conservación de las memorias acerca de las características de los cultivos: hábitats, agroecosistemas, prácticas de cultivo y posibilidades de consumo y comercialización.

El mortiño es un madero, me parece, como muy curioso, como bonito, una planta que tiene su misterio (...) Y hay veces como son palitos que salen derechitos, bonitos, entonces nosotros los cortábamos que para una zurriaga o hay veces que jugábamos con ellos que para cogerlos como de caballo, ponerlos en medio de las piernas, y así ¡móntese y vámonos! A veces de pequeño la utilizaba uno para eso. Mi papá sí para lo que le comento, como para madero o para sacar zurriagas o para un palito para un bastón. A mí se me hace curiosa esa planta mirarla, le encuentro como ese misterio, pero no sé por qué, se me hace bonita mirarla, le encuentro esa gracia. (Entrevista a habitante de la vereda Santa Bárbara, septiembre de 2018)

Por otro lado, las entrevistas permitieron reconocer que los conocimientos asociados con las plantas alimenticias están vinculados con el contexto histórico y de transmisión social del conocimiento, en esa medida, los cambios territoriales tienen un efecto en el cambio de relaciones con las plantas a través del tiempo.

Anteriormente se les utilizaba más, eran importantes y tenían un fin en la vida de nuestros antecesores en su dieta, ya que la guatila, los cornetos y el ají criollo era indispensable y de gran valor nutricional ya que antes era de más exigencia la fuerza del hombre en las labores del campo y el desgaste físico se evidenciaba más, las uvas y los mortiños, la consumían nuestros abuelos para los grandes viajes que debían realizar con mulas y cargas para llegar a los centro de comercialización, por ello es fundamental reconocer que estas especies han contribuido a lo largo de la historia alimentando nuestros indígenas y campesinos, para contribuir en el desarrollo de Bogotá. (Entrevista a habitante de la vereda Santa Bárbara, septiembre de 2018) 
Para mí es muy importante porque es la papa que nos dejaron nuestros abuelos, los indios, entonces hay que cuidar las cosas. Para mí es muy importante, el sabor y todo. Lo mismo esa papa, si uno no la cuida, se pierde, se acaba. Saber que es un producto que es bueno y, de todas maneras, económico. Es nativo, por eso uno trata de que no se acabe. (Entrevista a habitante de la vereda Curubital, agosto de 2018)

Según algunas conversaciones, la curiosidad por crear y movilizar conocimientos puede promover que las relaciones entre plantas y personas se renueven en estrategias que permitan regenerar la biodiversidad de la zona. En ese sentido, consideramos que no sólo documentar los resultados de la co-investigación, sino su proceso mismo, es una forma de reposicionar los sistemas de conocimiento local y de favorecer la renovación y recuperación del patrimonio biocultural bogotano.

Esta investigación permitió entender al alimento como vehículo que cohesiona sistemas bioculturales. Los conocimientos que persisten sobre las plantas en la Bogotá rural se han generado a partir de formas particulares de observar y transformar el entorno (Pérez y Matiz-Guerra, 2017). Así, estas plantas se relacionan con memorias de juegos, experiencias familiares, preparaciones, temporalidades y espacios que cobran vida en las experiencias de sus gentes. Para los investigadores locales, las plantas priorizadas eran buenas representantes de su diversidad biocultural. Según ellos, estas plantas "toda la vida han estado (...) desde los abuelos y ancestros", y que entonces "si estas plantas llegaran a desaparecer se destruiría el paisaje de Bogotá". Siendo parte de la cotidianidad de las personas de la Bogotá campesina, "siempre alguien tiene algo que contar de cada una de las plantas", con las que "se han desarrollado, recetas, saberes y una identidad propia del territorio" (Entrevista a habitante de la vereda Santa Bárbara, septiembre de 2018).

\section{b. El aprendizaje colectivo del primer encuentro culinario}

Quizá una de las relaciones más fuertes y cercanas entre las personas y las plantas involucran los procesos de preparación y consumo del alimento (Medina, 2017). Cocinar requiere compartir, implementar y descubrir prácticas y conocimientos que garanticen vivencias placenteras alrededor de la comida, y debido a lo experiencial de este proceso, usamos esa misma aproximación en esta investigación (Hernández, 2008). En la Tabla 2 se presentan las recetas que resultaron de los dos encuentros culinarios. El primero se enfocó en las prácticas locales, para lo que se ahondó en once preparaciones a partir de especies alimenticias de uso cotidiano. 
Esta selección se hizo en conjunto con las y los co-investigadores y algunos habitantes locales que participaron en el estudio. El segundo encuentro enriqueció la discusión sobre el valor patrimonial de las cocinas tradicionales al poner a dialogar las tradiciones culinarias de la ruralidad bogotana con las de otros territorios, promoviendo la búsqueda de nuevas preparaciones que, con base en técnicas e ingredientes tradicionales, incorporan un componente de innovación inspirado en las cocinas campesinas de la cordillera nariñense y la provincia del Tequendama, en Cundinamarca.

Tabla 2. Preparaciones asociadas a los dos encuentros culinarios desarrollados con la comunidad

\begin{tabular}{ll}
\hline I Encuentro culinario & II Encuentro culinario \\
\hline Yogurt de mora silvestre y mortiño & Maíz tostado con chochos y chicharrón \\
Chicha de ibias & Cornetos con guiso de maní \\
Jugo de mortiño & Locro de calabaza con habas, arvejas verdes, fríjol \\
Envueltos tres puntas & verde, mazorca, papa, chuguas y rebancá \\
Cubios guisados en leche fresca & Ensalada de guatila y pepino de \\
y mantequilla casera & guiso con hierbas silvestres \\
Cornetos guisados en leche fresca, & Conserva de tubérculos andinos y hierbas de páramo \\
suero y mantequilla casera & Ají de tomate de árbol \\
Rebancá con huevo & Ají de lulo de páramo \\
Pollo criollo con hierbas de páramo & Ají de maní \\
Ensalada de hierbas silvestres & Ají de mortiño \\
Ají criollo & Kumis patiano con frutos rojos de monte \\
Mermelada de mortiño con cuajada & (mora, morón, agraz, mortiño) \\
& Arequipe de papa \\
\hline
\end{tabular}

Fuente: elaboración propia.

En particular, el contacto con los ingredientes activó la memoria sensorial y permitió explorar la riqueza del universo material y simbólico asociado al alimento. Así, las conversaciones se extendieron más allá de los mismos ingredientes y preparaciones propuestas, integrando reflexiones sobre el territorio y su transformación, dejando en evidencia el carácter sistémico y biocultural de las cocinas tradicionales (Pimbert, 2017).

Las preparaciones reflejaron la creatividad de los habitantes para combinar ingredientes locales sin necesidad de una receta rigurosa, y desplegar lo aprendido a través de la transmisión colectiva de conocimientos culinarios (Figura 3). Además, esta experiencia dejó en evidencia que las cocinas tradicionales de la ruralidad de Bogotá son el resultado de un proceso histórico que no se limita a lo rural, sino que es definido por la relación con lo urbano (Jiménez et al., 2013). La disponibilidad de ingredientes facilitados por la cercanía a la ciudad, o las tendencias en la preparación de ciertos alimentos, convergen con la diversidad y 
disponibilidad de insumos locales, resultando en innovaciones culinarias que fusionan lo local con tendencias provenientes de distintas regiones representadas en los habitantes de la ciudad. Así, las cocinas tradicionales de la ruralidad son una manifestación de la multiculturalidad de Bogotá en sentido amplio.
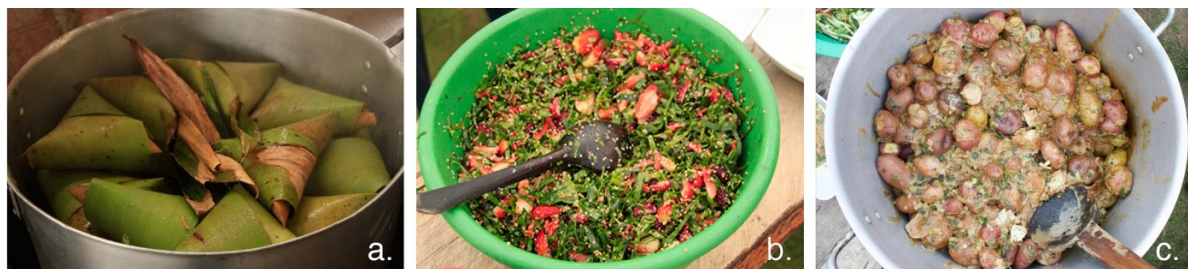

Figura 3. Algunas de las preparaciones realizadas durante los encuentros culinarios: envueltos tres puntas (a), ensalada de hierbas silvestres (b),

y cornetos con guiso de maní (c).

Fuente: fotos tomadas por los autores.

Preparaciones tradicionales emblemáticas, como los envueltos "tres puntas" (Figura 3a), son un reflejo de esta multiculturalidad de la cuenca media y alta del río Tunjuelo, cuyos ingredientes principales son el maíz y las hojas del "quiche", una especie de bromelia altoandina (Tillandsia sp.). En este caso, los ingredientes tienen orígenes diferentes: mientras que las hojas de quiche se "van a buscar al páramo" como dice un sabedor de la vereda Santa Bárbara, el maíz se trae de la ciudad, como ha ocurrido históricamente. Varios sabedores recuerdan que sus abuelos bajaban a la ciudad para abastecerse de lo que no producían en sus fincas, principalmente maíz y sal, y que estos viajes los hacían descalzos, pues las alpargatas las tenían reservadas para los días de misa. El contenido de los bultos con los que regresaban al hombro no sólo describe el tipo de intercambios que históricamente han existido entre lo rural y lo urbano, sino que también son el testimonio del vínculo entre territorios (Romero, 1992). Así, esta preparación entreteje diferentes relatos que hacen parte de la memoria colectiva de los habitantes de este territorio. Los envueltos son una preparación que, siendo tradicional, sigue dependiendo de lo que ofrecen tanto la ciudad como el campo, y que por esto mismo revela la porosidad de sus fronteras.

\section{c. Segundo encuentro culinario: innovación para la persistencia biocultural}

En el segundo encuentro, se conversó sobre la tradición y la innovación culinarias, destacando a la cocina como una manifestación del patrimonio cultural inmaterial y la diversidad biocultural, y la forma en 
que, al tener una naturaleza dinámica, la innovación y la tradición operan en ambos. El potencial de reactivación de la memoria y de renovación de la diversidad biocultural por medio del alimento tradicional fue el eje de estos diálogos. Adicionalmente, se discutió cómo las prácticas agrícolas actuales están siendo fuertemente influenciadas por la valoración económico-financiera, definida por el mercado, antes que la cultural. Para la comunidad había una percepción generalizada de que la pérdida de diversidad ha sido una tendencia continua en las últimas décadas, en especial en lo que se refiere a las especies asociadas tanto a las áreas boscosas como a las huertas (Ortiz et al., 2017).

Los participantes señalaron que esta pérdida también está relacionada con los patrones de consumo, donde la disponibilidad externa y la facilidad de acceso a ciertos ingredientes transforma las prácticas agrícolas, priorizando la comercialización sobre el autoconsumo (García-Molano, 2019). Estas dinámicas están estrechamente ligadas a las prácticas culinarias y cómo incluyen - o no- productos autóctonos de la región. Respecto a esto, aunque en los encuentros y las entrevistas realizadas muchos participantes defendieron la idea de que la comercialización debe pensarse como una instancia posterior a la de la recuperación y la reapropiación local de la diversidad agrícola, también se hizo evidente que la mayoría han dejado de cultivar y de consumir especies reconocidas por ellos mismos como "natales" y "lo más deliciosas" precisamente por no tener una salida comercial. Este es el caso de la "papa corneto" y otros tubérculos andinos:

El corneto, e igual con la guatila y el ají criollo, no encontramos que se esté rescatando, solo una vereda de las 14 de Usme lo está sembrando. Entonces uno dice, esperemos a ver, algo está pasando en el tema de que no sea comercial y que la gente dice que como no le da dinero y no tiene comercialización o salida entonces no lo siembra. Me decían que acá en la zona hay cinco variedades, pero solo hemos encontrado tres o dos. Sería interesante recuperarlas y si tenemos recetas el consumo podría aumentar. Cuando uno sabe de una receta, cuando uno sabe de eso, eso también es una motivación para sembrarlas. Pero qué bonito sería que las reprodujéramos nosotros. Eso ayudaría a que en unos años las sigamos conociendo y no se vuelva en una cosa de que sólo la conocí en un libro, en el papel. (Discusión durante el primer encuentro de cocina en la vereda El Hato, octubre de 2018)

Por ejemplo, los cornetos, cuando era niña pequeñita en la navidad se hacían los sancochos, los ajiacos y era con los cornetos, pero 
ya no existen. Cuando yo me casé yo me acuerdo que se hizo una chicha de ibias y eran, mejor dicho (...). (Discusión de cierre durante el II encuentro de cocina en la vereda El Hato, octubre de 2018)

(...) A nosotros se nos olvidó qué era guardar comida para nosotros y después sí para el mercado. (Discusión de cierre durante el I encuentro de cocina en la vereda El Hato, octubre de 2018)

Para algunos de los sabedores participantes, hablar del patrimonio cultural inmaterial de la ruralidad de Bogotá implicó considerar las tensiones entre la singularidad y la mutabilidad de la memoria biocultural. Por un lado, la singularidad, el arraigo y lo particular del patrimonio lo ancla a un territorio y a una cultura específicas y le otorga estabilidad. Por el otro, la permeabilidad, mutabilidad y relaciones con otros sistemas que influyen en los conocimientos y prácticas bioculturales las tornan inestables o vulnerables a transformarse e incluso a perderse (Barthel et al., 2013).

La tensión entre la tradición y la innovación no es necesariamente una contradicción y reconocerla permite complejizar la mirada sobre el patrimonio y comprender que este es cambiante, que está sujeto a presiones que pueden tener múltiples orígenes, y que su persistencia se asocia también a su capacidad de cambiar y ajustarse a un entorno en constante transformación (van der Hammen et al., 2009). En esta naturaleza dinámica del patrimonio residen posibilidades interesantes de innovación que pueden resultar en estrategias efectivas para su salvaguardia y permanencia, cuando se conservan dentro del contexto social y son trabajadas de forma autónoma por las comunidades.

"El patrimonio del campo también es de la ciudad" (palabras de una de las co-investigadoras en conversación personal, septiembre de 2018) y en esta misma dirección, los portadores del patrimonio agroalimentario y culinario de la ruralidad de Bogotá reconocen la singularidad de ciertas prácticas y saberes asociados a las cocinas tradicionales campesinas, pero también sostienen que éstas no existen de manera aislada al contexto de la Bogotá urbana.

Las cocinas tradicionales reflejan la co-evolución y co-dependencia de los contextos urbano y rural que constituyen tanto material como simbólicamente la identidad de la capital. Los intercambios entre estos dos contextos son los que han dado forma a las dinámicas regionales, aunque sea común que los procesos rurales -a pesar de abastecer de manera significativa a la ciudad-se subordinen a lo urbano. A pesar de esto, 
los habitantes de la Bogotá rural son conscientes de la importancia de su territorio en términos de su patrimonio cultural y natural, y reconocen su rol y responsabilidad como agricultores en la producción alimentaria diversa y en la protección de la biodiversidad. Las personas de la ruralidad también señalan la ventaja que tiene producir de forma autónoma en comparación con los habitantes de la ciudad, quienes dependen de terceros para acceder al alimento. En este sentido, muchos habitantes rurales comparten lo que expresa una sabedora de la vereda Santa Bárbara: "En un honor decir que soy campesina y en este territorio" (septiembre de 2018).

\section{d. "Volver a recordar esa imaginación"2: otros aspectos relacionados con el patrimonio culinario}

La cocina se aprende haciendo, y esta forma particular de transmisión de conocimientos no solo hace parte de las características que definen formalmente a las cocinas tradicionales de acuerdo con la Política para el conocimiento, la salvaguardia y el fomento de la alimentación y las cocinas tradicionales de Colombia (MinCultura, 2012), sino que además es sabiduría popular estrechamente ligada a las prácticas cotidianas. En el marco de los encuentros culinarios se resaltó que la cocina también se olvida si no se practica. Con esta perspectiva, muchos de los participantes y sabedores manifestaron el deseo de recuperar estas tradiciones $y$, en este sentido, sostuvieron que el espacio de encuentro había sido propicio para reactivar ese interés.

Me siento muy contenta de volver a los tiempos antiguos donde los papás las mamás le han enseñado a uno muchas cosas que uno ha dejado perder. (Discusión de cierre durante el II encuentro de cocina en la vereda El Hato, octubre de 2018)

Esto es una tradición de décadas y de décadas que nos han dejado (...) Eso lo hace a uno revivir, de verdad que es muy especial hacer este trabajo, el proceso de volver a hacer estos envueltos que hace tiempo no los preparábamos pero que no se nos ha olvidado. (Discusión de cierre durante el I encuentro de cocina en la vereda El Hato, octubre de 2018)

Al igual que otras manifestaciones de patrimonio cultural inmaterial y diversidad biocultural, las tradiciones culinarias son el resultado de un

Palabras de una sabedora durante la discusión de cierre del segundo encuentro de cocina en la vereda el Hato en Usme. 
largo proceso histórico y colectivo, lo que explica que estén atravesadas por sentimientos de pertenencia, identidad y permanencia histórica territorial (Denegri-Coria et al., 2016). Esto se refleja en las recetas que se seleccionaron para el primer taller, pues en ellas no solo estaban en juego los ingredientes y su preparación respectiva sino también la memoria biocultural de quienes han recibido y transmitido su legado.

Todo fue volverme a mi tierra. Recordar otra vez mi niñez, recordar la tierra, me sentí allá, eso fue una alegría. (Discusión de cierre durante el II encuentro de cocina en la vereda El Hato, octubre de 2018)

Además, la posibilidad de cocinar y comer juntos recordó a los campesinos de la ruralidad que las cocinas tradicionales son un medio para conocer y revivir nociones relativas a la experiencia material y simbólica de la vida en comunidad y del trabajo colectivo, que no solo son parte del origen de estas tradiciones, sino que también constituyen estrategias de salvaguarda:

Para mí esto fue una fiesta de familia (...) cocinar todos juntos, comer todos juntos, eso es lo que hacen las familias. Eso tenemos que tratarlo de guardar (...) también nos ayuda a recordar que podemos ir entre todos a recolectar, a cosechar, cocinar para compartir (...) no todo es el comercio, la plata, las cosas tienen un valor porque nos hacen personas, familia, comunidad, nos hacen disfrutar, nos hacen alegres. (Discusión de cierre durante el I encuentro de cocina en la vereda El Hato, octubre de 2018)

Otro aspecto clave de los encuentros fue que sirvieron como una plataforma para que los sabedores compartieran sus conocimientos y en el proceso mismo se dieran cuenta del enorme acervo de saberes y prácticas del que son portadores. Así mismo, el intercambio reveló la generosidad con la que están dispuestos a compartir estos conocimientos, lo que no solo habla de la forma como ellos los recibieron sino de cómo podrían transmitirlos.

Uno en estos talleres recuerda muchas cosas que las sabe hacer pero que no las practica (...) definitivamente hay que rescatar todas estas cositas porque uno come alimentos deliciosos y orgánicos. Me pareció muy importante, para mí fue algo muy provechoso compartir con todos ustedes (...) ahora pienso que lo más importante es no olvidar lo que nosotros aprendimos desde un comienzo y sobre todo seguir transmitiendo esos conocimientos. (Discusión de cierre durante el I encuentro de cocina en la vereda El Hato, octubre de 2018) 
Por último, la experiencia de los encuentros comprobó que este tipo de espacios son fundamentales para reactivar y recrear la memoria biocultural asociada a las tradiciones culinarias, la diversidad alimentaria y los vínculos del territorio, pero también para recordar y promover valores de la vida en comunidad. En otras palabras, los encuentros promovieron re-encuentros con el territorio en sentido amplio, es decir entre su gente y las otras formas de vida no humanas que lo constituyen. Como estrategia de reapropiación del patrimonio cultural inmaterial y de reactivación de la memoria biocultural, será importante que estas experiencias se retomen y se compartan con los habitantes de la ciudad. Contar con espacios que permitan dar a conocer lo propio y obtener a cambio un reconocimiento respecto a su valor fortalece las iniciativas de reapropiación.

\section{Conclusiones}

En esta investigación documentamos las prácticas relacionadas con las cocinas tradicionales de la ruralidad bogotana, promoviendo la reactivación de la memoria, las percepciones y los conocimientos asociados al alimento para facilitar la renovación de las relaciones bioculturales implicadas. Por esta razón, describimos en este artículo el proceso de co-investigación que promovió una experiencia colectiva y de reconocimiento mutuo entre sabedores locales, habitantes de la ruralidad y de la ciudad, e investigadores de la academia.

Al explorar distintas formas de abordar la forma en que el conocimiento y las prácticas sobre el alimento se transfieren y transforman, redescubrimos colectivamente la naturaleza dinámica de la memoria biocultural de la Bogotá rural. Sus habitantes han creado vínculos con las plantas basados en sus cotidianidades, en las condiciones ambientales, y en los distintos significados otorgados a las especies compañeras con quienes han diseñado sus formas de vida. Estos vínculos son tanto utilitarios como emocionales y estimulan la curiosidad sobre el funcionamiento del territorio y el registro de las relaciones bioculturales que conforman la cotidianidad local y regional. Aprender más sobre la biodiversidad y revitalizar las percepciones representa una oportunidad para reivindicar y revalorizar los vínculos sociales y culturales con el territorio.

Los sistemas de conocimiento derivados de estos procesos no están limitados al ámbito material y conservan una carga simbólica importante. Sin embargo, en su gran fluidez y alta velocidad de actualización, estos sistemas son muy sensibles a los cambios en el contexto social y ecológico donde se encuentran. Tanto lo que se conoce como la forma de conocer son fuertemente permeados por las relaciones de lo rural-urbano. 
Las prácticas culinarias y sus relaciones bioculturales son susceptibles de ser transformadas por formas de conocer en las que priman valores más utilitarios y desvinculantes derivados de la modernidad y de los mercados. El riesgo es que esa transformación implique una homogeneización de las dietas y de los paisajes que las acompañan, lo que significaría una pérdida de diversidad biológica y cultural.

Las metodologías de co-investigación nos permitieron abordar la pérdida de la memoria biocultural de forma colaborativa, incluyente y relevante para los habitantes de la Bogotá rural. Más aún, estas metodologías abrieron espacios para que los participantes diagnosticaran sus problemas y acudieran a su capacidad de indagación e innovación en búsqueda de alternativas. Así, los resultados de esta investigación se divulgaron en un recetario local que fue distribuido en las distintas veredas. Esta forma de socialización fue concertada con los participantes, buscando que les fuera de utilidad en procesos de empoderamiento, socialización y gestión local. En este recetario se hacen visibles las plantas asociadas a las recetas tradicionales y sus innovaciones, así como los conocimientos y saberes locales sobre los usos y preparaciones. Esperamos que sean un aporte para la recuperación, dinamización y salvaguarda del patrimonio biocultural asociado a las especies alimenticias de alta montaña.

No sólo es importante documentar la diversidad biocultural, también es urgente crear las condiciones para permitir que esta memoria se visibilice, se reactive y se renueve, y esto incluye brindar herramientas de protección al patrimonio material e inmaterial respecto a tendencias que motivan su detrimento, como la agresiva influencia de los mercados sobre la estabilidad de los medios de vida rurales y la presión del modelo actual de desarrollo que marginaliza la vida rural y la dignidad de sus habitantes. Proteger la diversidad biocultural implica reconocer y respetar a sus portadores y herederos, valorando sus formas de vida y sistemas de conocimiento que a lo largo de generaciones han enriquecido y revitalizado a los sistemas agroalimentarios. Pero también implica entender las transformaciones causadas por la porosidad de las fronteras urbano-rurales, y cómo pueden canalizarse hacia la renovación de las prácticas sin generar pérdidas irremediables de diversidad biocultural.

\section{Declaraciones}

Éticas:

Las personas entrevistadas son propietarios y tienen los derechos sobre su conocimiento tradicional aquí expuesto. Cualquier uso comercial 
de cualquiera de esta información requiere un consenso previo con los informantes involucrados y un acuerdo sobre la distribución de beneficios, dando cumplimiento del Protocolo de Nagoya sobre acceso y participación en los beneficios a las comunidades.

Contribución de los autores:

Todos los autores hicieron parte de la concepción, toma y análisis de datos, y escritura del documento.

Agradecimientos:

Agradecemos a las comunidades campesinas de la ruralidad de Bogotá quienes nos han acogido con tanta hospitalidad y receptividad, y nos han acompañado desde hace varios años. A los cuatro co-investigadores/as: Zonia, Rodrigo, Adriana y Erika, quienes fueron fundamentales en la formulación e implementación de la investigación. A Jennifer Rodríguez e Isabel Guevara por su participación activa durante los encuentros de cocina. Al Instituto Distrital de Patrimonio Cultural de Bogotá, Colombia, que financió este proyecto gracias a la beca de investigación "Campos, Mercados y Cocina".

\section{Referencias bibliográficas}

Andrade, L., Arrieta, M., Aya, D., Calambás, J., Castellanos, L., Chenut, P., Cortés, N., Cuellar, J. E., Cuervo, O. y Franco, G. C. (2018). Fuentes vivas en el borde: investigación y experiencias colaborativas para la gobernanza de un sur sostenible en Bogotá. Universidad Externado de Colombia.

Barthel, S., Crumley, C. L. y Svedin, U. (2013). Biocultural Refugia: Combating the Erosion of Diversity in Landscapes of Food Production. Ecology and Society, 18(4), 71. http://dx.doi.org/10.5751/ES-06207-180471

Benz, B. F., Cevallos, J. E., Santana, F. M., Rosales, J. A. y Graf, S. M. (2000). Losing Knowledge about Plant Use in the Sierra de Manantlan Biosphere Reserve, Mexico. Economic Botany, 54(2), 183-191. https://doi.org/10.1007/BF02907821

Bonilla-Bolaños, A. G. y Singaña-Tapia, D. A. (2019). La productividad agrícola más allá del rendimiento por hectárea: análisis de los cultivos de arroz y maíz duro en Ecuador. La Granja: Revista de ciencias de la vida, 29(1), 70-83. http://doi.org/10.17163/lgr.n29.2019.06

Bowman, M. S. y Zilberman, D. (2013). Economic Factors Affecting Diversified Farming Systems. Ecology and Society, 18(1), 33. http://dx.doi.org/10.5751/ES-05574-180133

Cabrera-Serrano, D. C. (2005). Identidad y globalización: encuentros y transformaciones entre las cocinas nacionales. Universitas Humanistica, 60, 85-93. https://revistas. javeriana.edu.co/index.php/univhumanistica/article/view/9491/7695 
Clavijo-Ponce, N. L. y Pérez-Martínez, M. E. (2014). Tubérculos andinos y conocimiento agrícola local en comunidades rurales de Ecuador y Colombia. Cuadernos de Desarrollo Rural, 11(74), 149-166. https://doi.org/10.11144/Javeriana.CRD11-74.taca

Consuegra, C., Ortiz, S. y Pérez, D. (2017). Recetas natales del campo bogotano. Bogotá, Colombia: Instituto Distrital de Patrimonio Cultural. https://www.researchgate.net/ publication/326400877_Recetas_natales_del_campo_bogotano

Delgado, F. y Escobar, E. (2006). El diálogo intra, intercultural e intercientífico en el contexto global y latinoamericano para un desarrollo endógeno sostenible. Diálogo intercultural e intercientífico. Plural Editores.

Denegri-Coria, M., Knabe-Álvarez, Y., Sepúlveda-Maldonado, J., Orellana, L., Ruiz, O., Vivallo, O. y Schnettler-Morales, B. (2016). Socialización y transmisión intergeneracional en pautas del consumo de alimentos: su impacto en la identidad cultural alimentaria en mujeres Mapuche urbanas de Chile. Ra Ximhai, 12(4), 57-70. https://www.redalyc. org/pdf/461/46146927004.pdf

Ellis, E. C., Antill, E. C. y Kreft, H. (2012). All Is Not Loss: Plant Biodiversity in the Anthropocene. PLoS ONE, 7(1), e30535. 2. https://doi.org/10.1371/journal.pone.0030535

Fals-Borda, O. (1999). Orígenes universales y retos actuales de la IAP (Investigación Acción Participativa). Análisis político, (38), 73-90.

Fu, Y., Chen, J., Guo, H., Hu, H., Chen, A. y Cui, J. (2010). Agrobiodiversity loss and livelihood vulnerability as a consequence of converting from subsistence farming systems to commercial plantation-dominated systems in Xishuangbanna, Yunnan, China: A household level analysis. Land Degradation \& Development, 21(3), 274-284. https:// doi.org/10.1002/ldr.974

García-Molano, O. J. (2019). Cocinas tradicionales en declive: riesgo para el patrimonio cultural y la seguridad alimentaria en Guayatá. Revista de Antropología y Sociología: Virajes, 21(2), 15-38. http://vip.ucaldas.edu.co/virajes/downloads/Virajes21(2)_2.pdf

Gómez-Baggethun, E. (2009). Perspectivas del conocimiento ecológico local ante el proceso de globalización. Papeles de relaciones ecosociales y cambio global, 107, 57-67.

Haraway, D. (2003). The companion species manifesto: Dogs, people, and significant otherness. Prickly Paradigm Press Chicago.

Harding, S. G. (1994). Is science multicultural?: Challenges, resources, opportunities, uncertainties. Configurations, 2(2), 301-330.

Hernández, M. (2008). A la altura de lo cotidiano: algunos sentidos del cocinar y comer en casa. Fundamentos en Humanidades, 9(18), 71-89. http://www.redalyc.org/articulo. oa?id=18411970004

Jiménez, J. A., Salamanca, D. F. y López, D. P. (2013). Territorio y construcción de subjetividades en Bogotá. Transferencias entre lo rural y lo urbano en las localidades de Chapinero, Usme y Ciudad Bolívar. Investigaciones en construcción, 28, 167-210.

Laurance, W. F., Nascimento, H. E. M., Laurance, S. G., Andrade, A., Ribeiro, J., Giraldo, J. P., Lovejoy, T. E., Condit, R., Chave, J. y Harms, K. E. (2006). Rapid decay of treecommunity composition in Amazonian forest fragments. Proceedings of the National Academy of Sciences, 103(50), 19010-19014. https://doi.org/10.1073/pnas.0609048103 
Medina, F. X. (2017). Reflexiones sobre el patrimonio y la alimentación desde las perspectivas cultural y turística. Anales de Antropología, 51(2), 106-113. https://doi.org/10.1016/j. antro.2017.02.001

Ministerio de Cultura de Colombia. (2012). Política para el conocimiento, la salvaguardia y el fomento de la alimentación y las cocinas tradicionales de Colombia. Ministerio de Cultura de Colombia.

Montenegro de Wit, M. (2016). Are we losing diversity? Navigating ecological, political, and epistemic dimensions of agrobiodiversity conservation. Agriculture and Human Values, 33, 625-640. https://doi.org/10.1007/s10460-015-9642-7

Nazarea-Sandoval, V. D. (1998). Cultural memory and biodiversity. University of Arizona Press.

Ortiz, S., Consuegra, C., van der Hammen, M. C. y Pérez, D. (2021). Perspectivas urbanorurales sobre la circulación de dos frutos silvestres del bosque altoandino en sistemas agroalimentarios de Bogotá, Colombia. Revista Etnobiología, 19(1), 81-95. https:// revistaetnobiologia.mx/index.php/etno/article/view/397

Ortiz, S., De la Pava, R. y Quiroga, C. (2017). Agrobiodiversidad y cultura: un análisis desde el lugar y los agroecosistemas en la cuenca alta del río Tunjuelo en Bogotá. Entorno Geográfico, (13), 24-41. https://doi.org/10.25100/eg.v0i13.6029

Pérez-Martínez, M. E. (2011). La adaptabilidad de pobladores y asentamientos rurales en áreas de conurbación: El caso de la ciudad de Bogotá (Colombia). Cuadernos de Desarrollo Rural, 5(60), 61-86. https://revistas.javeriana.edu.co/index.php/desarrolloRural/ article/view/1207

Pérez, D. y Matiz-Guerra, L. C. (2017). Uso de las plantas por comunidades campesinas en la ruralidad de Bogotá D.C. Caldasia, 39(1), 68-78. https://doi.org/10.15446/caldasia. v39n1.59932

Perreault, T. (2005). Why chacras (swidden gardens) persist: agrobiodiversity, food security, and cultural identity in the Ecuadorian Amazon. Human Organization, 64(4), 327-339. https://doi.org/10.17730/humo.64.4.e6tymmka388rmybt

Perrault-Archamault, M. y Coomes, O. T. (2008). Distribution of agrobiodiversity in home gardens along the Corrientes River, Peruvian Amazon. Economic Botany, 62(2), 109126. https://doi.org/10.1007/s12231-008-9010-2

Pimbert, M. (2017). Democratizing knowledge and ways of knowing for food sovereignty, agroecology, and biocultural diversity. En M. Pimbert (ed.), Food Sovereignty, Agroecology and Biocultural Diversity (pp. 259-321). Taylor \& Francis.

Polk, M. (2015). Transdisciplinary co-production: Designing and testing a transdisciplinary research framework for societal problem solving. Futures, 65, 110-122. https://doi. org/10.1016/j.futures.2014.11.001

Ramirez, C. (2007). Ethnobotany and the loss of traditional knowledge in the 21st century. Ethnobotany Research and Applications, 5, 245-247. https://doi.org/10.17348/ERA. 5.0.245-247

Reyes-García, V., Vadez, V., Byron, E., Apaza, L., Leonard, W. R., Perez, E. y Wilkie, D. (2005). Market Economy and the Loss of Folk Knowledge of Plant Uses: Estimates from the Tsimane' of the Bolivian Amazon. Current Anthropology, 46(4), 651-656. https://doi. org/10.1086/432777 
Reyes-García, V., Guèze, M., Luz, A. C., Paneque-Gálvez, J., Macía, M. J., Orta-Martínez, M., Pino, J. y Rubio-Campillo, X. (2013). Evidence of traditional knowledge loss among a contemporary indigenous society. Evolution and Human Behavior, 34(4), 249-257. https://doi.org/10.1016/j.evolhumbehav.2013.03.002

Rhoades, R. E. y Nazarea, V. D. (1999). Local management of biodiversity in traditional agroecosystems. En W. W. Collins y C. O. Qualset (eds.), Biodiversity in agroecosystems (pp. 215-236). Lewis Publishers.

Rodríguez-Calderón, A., Muñoz, J. A., Moreno, D. y Celis, M. (2019). Describing and diffusing the ethnobotanical knowledge of Bogotá D.C. (Colombia) through an online tool focused on common names of plants. Acta Botanica Brasilica, 33(2), 303-314. http://dx.doi.org/10.1590/0102-33062018abb0337

Romero, M. E. (1992). Traditional recipes of migrants from Boyacá to Bogotá, Colombia. Ecology of Food and Nutrition, 27, 189-198. https://doi.org/10.1080/03670244.1992. 9991243

Thrupp, L. A. (2002). Linking agricultural biodiversity and food security: the valuable role of agrobiodiversity for sustainable agriculture. International Affairs, 76(2), 265-281. https://doi.org/10.1111/1468-2346.00133

Toledo, V. y Barrera-Bassols, N. (2014). La memoria biocultural. La importancia ecológica de las sabidurías tradicionales. Universidad del Cauca.

Torres-Pabón, G. (2020). Balance introductorio de la literatura sobre la relación entre condiciones socioeconómicas y consumo de alimentos. Revista Colombiana de Sociología, 43(2). https://doi.org/10.15446/rcs.v43n2.78453

Tsuji, L. J. S. (1996). Loss of cree traditional ecological knowledge in the Western James Bay region of northern Ontario, Canada: a case study of the sharp-tailed grouse, Tympanuchus phasianellus. The Canadian Journal of Native Studies, 16(2), 283-292.

Vandebroek, I. y Voeks, R. (2019). The Gradual Loss of African Indigenous Vegetables in Tropical America: A Review. Economic Botany, 72(4), 543-571. https://doi.org/10.1007/ s12231-019-09446-3

Van der Hammen, M. C., Lulle, T. y Palacio, D. C. (2009). La construcción del patrimonio como lugar: un estudio de caso en Bogotá. Antípoda, (8), 61-85. https://doi.org/10.7440/ antipoda8.2009.03

Voeks, R. y Leony, A. (2004). Forgetting the Forest: Assessing Medicinal Plant Erosion in Eastern Brazil. Economic Botany, 58, S294-S306. https://doi.org/10.1663/00130001(2004)58[S294:FTFAMP]2.0.CO;2

Wolff, F. (2004). Industrial transformation and agriculture: Agrobiodiversity loss as sustainability problem. En J. Jacob, M. Binder y A. Wieczorek (eds.), Governance for industrial transformation: Proceedings of the 2003 Berlin conference on the human dimensions of global environmental change (pp. 338-355). University of Berlin.

Zimmerer, K. S. (2004). Cultural ecology: placing households in human-environment studies the cases of tropical forest transitions and agrobiodiversity change. Progress in Human Geography, 28(6), 795-806.https://doi.org/10.1191\%2F0309132504ph520pr 